\title{
Relación del autoconcepto con la condición física y la composición corporal en una muestra de adolescentes
}

\author{
Rafael E. Reigal-Garrido ${ }^{1 *}$, Carlos A. Becerra-Fernández ${ }^{2}$, Antonio Hernández-Mendo ${ }^{2}$ e Ignacio Martín-Tamayo ${ }^{1}$ \\ 1 Universidad de Granada (España) \\ 2 Universidad de Málaga (España)
}

Resumen: El propósito de este trabajo es examinar las relaciones entre el autoconcepto y diferentes variables de la condición física, así como el porcentaje de masa grasa. Han participado en el estudio 283 adolescentes de la ciudad de Málaga (España), en edades comprendidas entre los 14 y 16 años $(M=15.14 ; D T=.76)$. Se ha utilizado el Cuestionario Autoconcepto Forma 5 (AF5) para evaluar el autoconcepto multidimensional (académico, social, emocional, familiar y físico). Se ha medido el porcentaje de masa grasa corporal usando un instrumento de bioimpedancia eléctrica y el consumo máximo de oxígeno a través del test de Course Navette. Además, se ha estimado el tiempo en recorrer 50 metros lisos, la distancia alcanzada mediante salto horizontal y la amplitud de movimiento con la prueba flexión profunda de tronco. Los análisis de correlación y regresión lineal efectuados ponen de manifiesto la conexión entre las variables objeto de estudio, siendo el porcentaje de masa grasa y el consumo de oxígeno máximo aquellas que mejor se han relacionado con el autoconcepto. Además, las dimensiones física y emocional son las que han sido predichas con un mayor porcentaje de la varianza explicada.

Palabras clave: autoconcepto; adolescencia; condición física; composición corporal.

\section{Introducción}

Son numerosas las evidencias que ponen de relieve el impacto que tienen los estilos de vida activos sobre la salud, tanto a nivel físico como psicológico o social (Annesi, 2010; Goñi e Infante, 2010; Pontifex et al., 2011). Además, varios estudios señalan que los riesgos de morbimortalidad en edad adulta se relacionan con la condición física en etapas tempranas. De hecho, aunque las manifestaciones clínicas de diversas enfermedades puedan presentarse posteriormente, el origen de dichas patologías se encuentra en factores existentes en la infancia y la adolescencia. Entre ellos, la condición física y la composición corporal son algunas de las variables que pueden incidir en los estados de salud futuros, por lo que el ejercicio físico regular y el control de la obesidad son algunos de los aspectos sobre los que hay que intervenir adecuadamente (Eisenmann, Wickel, Welk y Blair, 2005; Ferreira et al., 2005).

El análisis de la actividad física debe contemplarse teniendo en cuenta el grado de condición física alcanzada, existiendo estudios que lo asocian a una mejor valoración del impacto sobre la salud. La relación entre ambas no siempre supone un dato fiable del estado del forma física, lo cual debe ser evaluado a través de procedimientos directos (Ramírez-Lechuga, Femia, Sánchez-Muñoz y Zabala, 2011). En este sentido, García-Artero et al. (2007) señalan que una mejor

* Dirección para correspondencia [Correspondence address]: Rafael Enrique Reigal Garrido. Grupo Investigación CTS-642 (IDAFISAD), Facultad de Ciencias de la Actividad Física y el Deporte, Universidad de Granada. Carretera de Alfacar s/n, 18011, Granada (España). Email: rafareigal@gmail.com
Title: Relationships of self-concept with physical fitness and body composition in a sample of adolescents.

Abstract: The aim of this study is to analyse the relationship between selfconcept and several variables of physical fitness, as well as the body fat percentage. The participants in the study were 283 teenagers from the city of Malaga (Spain), whose age ranged between 14 and 16 years old ( $M=$ 15.14; SD = .76). The "Autoconcepto Forma 5" questionnaire (AF5) was used to evaluate the multidimensional self-concept (academic, social, emotional, physical, and family self-concept). Moreover, the body fat percentage was measured by means of a bioelectrical impedance analysis. The Course-Navette test was used to assess the maximal oxygen consumption. Other measured variables were the time to run 50 metres, the distance jumped horizontally, and the range of movement (by means of a deep trunk flexion test). The linear regression and correlation analysis highlighting that the variables which have the strongest association with selfconcept are the body fat percentage and the maximal oxygen consumption. Besides, physical and emotional dimensions are those that have been predicted with a higher percentage of explained variance.

Key words: self concept; adolescence; physical fitness; body composition.

capacidad aeróbica y una mayor fuerza muscular se asocia con un perfil lipídico-metabólico más saludable, tras un estudio realizado con adolescentes españoles. En él, se pone de manifiesto que valores del consumo máximo de oxígeno por encima de $51.6 \mathrm{ml} \cdot \mathrm{kg}^{-1} \cdot \mathrm{min}^{-1}$ en chicos y $42.2 \mathrm{ml} \cdot \mathrm{kg}^{-}$ ${ }^{1} \cdot \mathrm{min}^{-1}$ en chicas supone un menor riesgo de padecer enfermedades cardiovasculares. Martins et al. (2010) también encuentra evidencias, en una investigación con adolescentes portugueses, según las cuales un mayor rendimiento cardiovascular disminuye el riesgo de patologías cardiacas.

Asimismo, un buen nivel de condición física está relacionado con beneficios sobre otras variables, como son las de tipo psicosocial o cognitivo. Por ejemplo, Chaddock et al. (2012) señalan, en un estudio con niños norteamericanos, la importancia que posee la condición física en edades tempranas sobre el rendimiento cognitivo. Indican que la capacidad aeróbica influye en dichas habilidades, pudiéndose predecir el grado de desarrollo un año más tarde. A su vez, Mota et al. (2012), en un estudio con adolescentes portuguesas, obtienen resultados que indican mejores percepciones de salud en aquellas que tenían un mejor rendimiento cardiorrespiratorio y una composición corporal más saludable. Por su parte, Dunton, Schneider y Cooper (2007), en un trabajo con adolescentes norteamericanas, señalan que las mejoras en la capacidad aeróbica están relacionadas con una percepción más positiva de la autoeficacia general.

Cuando se analiza el estado físico de una persona, en numerosas ocasiones se ha puesto de manifiesto la importancia de la composición corporal en la valoración de la salud. Ruiz et al. (2009), tras revisar diferentes investigaciones sobre población infantil y adolescente, ponen de relieve me- 
jores perfiles cardiovasculares y estado de salud de la espalda cuando los porcentajes de masa grasa y magra se sitúan dentro del umbral de salud. Por otro lado, Padilla-Moledo et al. (2012) observan, en una muestra de niños y adolescentes españoles, que cuando se aumenta la capacidad cardiorrespiratoria y disminuye el porcentaje de grasa corporal mejoran las valoraciones que hacen de su calidad de vida, estando más satisfechos con ella y mostrando percepciones de salud más positivas. Un estudio llevado a cabo por Franklin, Denyer, Steinbeck, Caterson y Hill (2006), con un grupo de niños y adolescentes australianos, pone de manifiesto que la composición corporal afecta a la percepción de autoestima, existiendo una peor valoración si son obesos o tienden a ello.

Por otro lado, el concepto que tienen las personas de sí mismas es uno de los factores que influyen en el desarrollo de los adolescentes, asociándose a un mejor funcionamiento personal y social, el cual ha sido estudiado en numerosas ocasiones (Esnaola, Goñi y Madariaga, 2008; Moreno, Moreno y Cervelló, 2009). Está implicado en la relación que tiene la persona con su entorno, favoreciendo la adopción de conductas saludables y determinando el éxito que puede alcanzar en ámbitos tan importantes como el académico, social o deportivo (Guillén y Ramírez, 2011; Slutzky y Simpkins, 2009). Actualmente, el autoconcepto se entiende como una realidad de naturaleza multidimensional y jerárquica, existiendo una dimensión más general y estable que se sitúa sobre otras más específicas en niveles intermedios e inferiores, como el autoconcepto físico, emocional, familiar, académico o social (Esnaola, Rodríguez y Goñi, 2011; Shavelson, Hubner y Stanton, 1976).

Diversos investigadores han indagado en la relación de la condición física y la composición corporal con el autoconcepto. Crocker, Sabiston, Kowalski, McDonough y Kowalski (2006) realizan un estudio sobre adolescentes canadienses y encuentran que el índice de masa corporal está relacionado con las percepciones de autoconcepto general y físico. Castillo y Molina-García (2009) estudian a un grupo de universitarios españoles encontrando que aquellos que tienen un mayor porcentaje de grasa corporal poseen una peor percepción de la competencia personal, así como menor autoestima. Una investigación llevada a cabo por Velez, Golem y Arent (2010), sobre un grupo de adolescentes norteamericanos, indica que un programa de actividad física, basado en entrenamiento de resistencia, disminuye los porcentajes de masa grasa y provoca una mejora en los niveles de autoconcepto físico y general.

En esta línea, Strong et al. (2005), tras realizar una amplia revisión sobre estudios cuyos participantes estaban en edad escolar, ponen de manifiesto que la relación entre la práctica de actividad física y el autoconcepto, tanto general como específico, está condicionada por el tipo de actividad llevada a cabo, como la encaminada a mejorar la capacidad aeróbica, fuerza o flexibilidad. Por su parte, Carraro, Scarpa y Ventura (2010) analizan a un grupo de adolescentes italianos y encuentran que la condición física está asociada a mejores puntuaciones en las percepciones de autoconcepto físico. Por otro lado, Guillén y Ramírez (2011) realizan un estudio con un grupo de niños españoles, con edades entre 10 y 13 años, encontrando asociaciones significativas entre medidas de condición física y algunas dimensiones del autoconcepto como la conductual o la intelectual.

En base a los antecedentes descritos, la presente investigación analiza las relaciones entre el autoconcepto multidimensional, los resultados obtenidos en diferentes pruebas de condición física y el porcentaje de grasa corporal. El objetivo principal es determinar si el porcentaje de masa grasa y la condición física pueden ser predictores óptimos del autoconcepto en una muestra de adolescentes.

\section{Método}

Se ha utilizado metodología correlacional de tipo transversal, usando la encuesta y la evaluación de la condición física como herramientas para la toma de datos (Cubo, 2011; Martín, 2011).

\section{Participantes}

Los participantes de este estudio han sido 309 adolescentes de la ciudad de Málaga (España), los cuales pertenecen a los cursos de $3^{\circ}$ y $4^{\circ}$ de ESO. Se han eliminado 26 por no cumplimentar adecuadamente el cuestionario, dejando un alto porcentaje de ítems sin rellenar, o por obtener puntuaciones muy desviadas (outliers). De los 283 restantes, el $45.94 \%$ son chicos $(n=130)$ y el $54.06 \%$ chicas $(n=153)$, con edades comprendidas entre los 14 y 16 años $(M=15.14 ; D T=$ $.76)$.

\section{Instrumentos}

a) Cuestionario Autoconcepto Forma 5 (AF5- García y Musitu, 2001). Este cuestionario, formado por 30 ítems, evalúa el autoconcepto multidimensional, estando formado por 5 factores: académico/profesional (ítems 1, 6, 11, 16, 21 y 26), social (ítems 2, 7, 12, 17, 22 y 27), emocional (ítems 3, 8, 13, 18, 23 y 28), familiar (ítems 4, 9, 14, 19, 24 y 29) y físico (ítems $5,10,15,20,25$ y 30). Se contesta mediante una escala con puntuaciones entre 1 y 99 , en el que 1 significa estar en total desacuerdo y 99 un acuerdo máximo con lo dictado en cada cuestión. Posteriormente esta escala se traslada a una más reducida con puntuaciones entre 1 y 10 para su análisis e interpretación. La aplicación de este cuestionario se ubica en un espectro de población muy amplio, desde la infancia hasta la edad adulta. Los análisis de fiabilidad (Alfa de Cronbach), en este trabajo y para cada subescala, ofrecen puntuaciones adecuadas (entre .71 - .89).

b) Evaluación de la condición física. Se han utilizado varias pruebas de la batería EUROFIT (1993), como el test de Course Navette, para calcular de forma indirecta el consumo máximo de oxígeno (VO2max), y el test de salto horizontal para valorar la fuerza explosiva en el miembro 
inferior. Además, se han realizado otras como la carrera de 50 metros para evaluar la velocidad y la flexión profunda de tronco para estimar la amplitud de movimiento (Martínez, 2011).

c) Composición corporal: se ha empleado un bioimpedanciómetro (Tanita ${ }^{\circledR}$ Body Composition Monitor modelo BC543) para medir el porcentaje de masa grasa.

\section{Procedimiento}

El muestreo ha sido polietápico y por conglomerados de los centros educativos de la ciudad de Málaga. Se ha solicitado el consentimiento informado, indicando que los datos serían anónimos y la participación voluntaria. En clase de Educación Física se ha evaluado la condición física y se han tomado medidas de composición corporal. Para que los resultados obtenidos con la Tanita ${ }^{\circledR}$ BC-543 sean fiables y tengan un mínimo margen de error, se han considerado las pautas y recomendaciones propuestas por George, Fisher y Vehrs (2007) para la estimación de la composición corporal. El protocolo a seguir ha sido: evitar ejercicios agotadores el día anterior, no alterar significativamente la alimentación el día previo a la prueba, vestir ropa cómoda, controlar la toma de medicamentos que puedan alterar los niveles de agua corporal y no retener líquidos. Por otro lado, el cuestionario AF5 se ha rellenado de forma colectiva, dado que es autoadministrable, siendo explicado adecuadamente y estando presente un colaborador para resolver posibles dudas.

\section{Análisis de los datos}

Se han realizado análisis descriptivos e inferenciales. Para analizar las correlaciones entre el autoconcepto, la condición física y la composición corporal se ha utilizado el coeficiente bivariado de Pearson. La capacidad predictiva de la condición física y el porcentaje de masa grasa sobre la percepción de concepto personal se ha evaluado mediante análisis de regresión lineal. Para el procesamiento estadístico de los datos se ha usado el programa informatizado SPSS en su versión 20.0 .

\section{Resultados}

Las tablas 1 y 2 muestran los estadísticos descriptivos de las variables objeto de estudio, así como las correlaciones (Pearson) establecidas entre ellas.

Tabla 1. Estadísticos descriptivos.

\begin{tabular}{|c|c|c|c|c|c|c|}
\hline & Mínimo & Máximo & Media & $D T$ & Asimetría & Curtosis \\
\hline Masa grasa $(\%)$ & 7.00 & 37.70 & 20.19 & 7.26 & .05 & -.85 \\
\hline $\mathrm{VO} 2 \max \left(\mathrm{ml} \cdot \mathrm{kg}^{-1} \cdot \mathrm{min}^{-1}\right)$ & 27.80 & 57.12 & 43.44 & 7.37 & .06 & -.80 \\
\hline Test velocidad $50 \mathrm{~m}$. (seg) & 6.69 & 10.77 & 8.24 & .93 & .53 & -.18 \\
\hline Salto horizontal (cm) & 87 & 249 & 169.95 & 36.00 & .28 & -.77 \\
\hline Flexión profunda $(\mathrm{cm})$ & 6 & 43 & 22.62 & 8.13 & .20 & -.29 \\
\hline Autoconcepto académico & 2.23 & 9.77 & 6.49 & 1.73 & -.54 & -.51 \\
\hline Autoconcepto social & 3.67 & 9.85 & 7.53 & 1.50 & -.83 & -.16 \\
\hline Autoconcepto emocional & 3.32 & 9.57 & 6.58 & 1.43 & -.06 & -.98 \\
\hline Autoconcepto familiar & 4.78 & 9.90 & 8.14 & 1.36 & -.80 & -.19 \\
\hline Autoconcepto físico & 1.68 & 9.48 & 6.20 & 1.78 & -.30 & -.66 \\
\hline
\end{tabular}

VO2max = consumo máximo oxígeno

Tabla 2. Nivel de correlación (Pearson).

\begin{tabular}{|c|c|c|c|c|c|c|c|c|c|}
\hline \multirow[b]{3}{*}{ Porcentaje masa grasa } & \multicolumn{9}{|c|}{ Autoconcepto } \\
\hline & \multicolumn{2}{|c|}{ Académico } & \multicolumn{2}{|c|}{ Social } & \multicolumn{2}{|c|}{ Emocional } & \multirow{2}{*}{$\begin{array}{l}\text { Familiar } \\
.03\end{array}$} & \multicolumn{2}{|c|}{ Físico } \\
\hline & -.11 & & -.26 & $* * *$ & -.42 & $* * *$ & & -.59 & *** \\
\hline Consumo máximo oxígeno & .25 & *** & .23 & $* * *$ & .44 & $* * *$ & -.12 & .51 & $* * *$ \\
\hline Test velocidad 50 metros & .01 & & -.13 & $*$ & -.37 & $* * *$ & .07 & -.55 & $* * *$ \\
\hline Salto horizontal & .05 & & .05 & & .37 & $* * *$ & -.05 & .55 & $* * *$ \\
\hline Flexión profunda tronco & .25 & $* *$ & .25 & $* * *$ & .03 & & .08 & .29 & $* * *$ \\
\hline
\end{tabular}

$* p<.05 ; * * p<.01 ; * * * p<.001$

Los análisis de correlación efectuados indican la existencia de relaciones significativas entre los valores del porcentaje de masa grasa y la condición física con los diferentes factores del Cuestionario Autoconcepto Forma 5. Las asociaciones más altas se establecen con las puntuaciones de la subescala referida al autoconcepto físico. Respecto a las demás, las correlaciones más elevadas se producen entre el porcentaje de masa grasa y el autoconcepto emocional, así como entre consumo máximo de oxígeno y el autoconcepto emocional (tabla 2), siendo correlaciones de nivel moderado en función de lo establecido por Cohen (1988).

En la tabla 3 se muestran los análisis de regresión lineal efectuados (utilizando la técnica de pasos sucesivos), salvo el referido al autoconcepto familiar por no ofrecer resultados satisfactorios. Los análisis cumplen el supuesto de linealidad en la relación entre variables predictoras y criterio, así como los 
de homocedasticidad y distribución normal de los residuos, cuyo valor medio es 0 y la desviación típica prácticamente 1 (.99). Además, los valores de Durbin-Watson son adecuados, dado que se encuentran en un rango entre 1.77 y 2.01. Pardo y Ruiz (2005) consideran que cuando el estadístico se encuentra entre 1.5 y 2.5 se puede asumir que los residuos son independientes, cumpliéndose el supuesto de independencia de las variables independientes con respecto a la dependiente. Por otro lado, los estadísticos de colinealidad indican valores aceptables de inflación de la varianza y del índice de Tolerancia (Hair, Anderson, Tatham y Black, 1999).

El análisis efectuado para el autoconcepto académico no incluye la variable porcentaje de masa grasa y el tiempo en recorrer 50 metros por falta de significación $(p>.05)$ y se ha excluido el salto horizontal por presentar indicios de colinealidad, con valores de tolerancia bajos (.19) y altos de inflación de la varianza (5.20), acompañado por cambio de signo entre las correlaciones de orden cero, parcial y semiparcial. El análisis realizado sobre el autoconcepto social excluye, en primer lugar, al salto horizontal, por tener valores bajos de tolerancia (.19) y altos de inflación de la varianza (5.37), así como cambio de signo entre las correlaciones. En segundo lugar, al consumo máximo de oxígeno y el tiempo en recorrer 50 metros por falta de significación $(\phi>.05)$. El análisis sobre el autoconcepto emocional excluye a las variables tiempo en recorrer 50 metros, salto horizontal y flexión profunda de tronco ( $\mathrm{p}>.05)$. Por último, el análisis realizado sobre el autoconcepto físico no incluye las variables salto horizontal y flexión profunda de tronco $(p>.05)$.

Tabla 3. Análisis de regresión lineal (pasos sucesivos).

\begin{tabular}{|c|c|c|c|c|c|c|c|c|}
\hline Variable criterio & Variables Predictoras & $R$ & $\mathrm{R}^{2}$ corregida & $D-W$ & Beta & $\mathrm{t}$ & $\mathrm{T}$ & FIV \\
\hline \multirow[t]{3}{*}{ Autoconcepto académico } & & .31 & .09 & 1.77 & & & & \\
\hline & VO2max & & & & .197 & $3.33 * *$ & .93 & 1.08 \\
\hline & FPT & & & & .187 & $3.16^{* *}$ & .93 & 1.08 \\
\hline \multirow[t]{3}{*}{ Autoconcepto social } & & .30 & .09 & 2.01 & & & & \\
\hline & $\% \mathrm{MG}$ & & & & -.188 & $-3.02 * *$ & .84 & 1.19 \\
\hline & FPT & & & & .176 & $2.83^{* *}$ & .84 & 1.19 \\
\hline \multirow[t]{3}{*}{ Autoconcepto emocional } & & .47 & .22 & 1.89 & & & & \\
\hline & $\mathrm{VO} 2 \max$ & & & & .293 & $4.13^{* * *}$ & .55 & 1.81 \\
\hline & $\% \mathrm{MG}$ & & & & -.219 & $-3.09 * *$ & .55 & 1.81 \\
\hline \multirow[t]{4}{*}{ Autoconcepto físico } & & .62 & .39 & 1.99 & & & & \\
\hline & $\% \mathrm{MG}$ & & & & -.333 & $-4.52 * * *$ & .40 & 2.48 \\
\hline & Test $50 \mathrm{~m}$. & & & & -.214 & $-3.02 * *$ & .44 & 2.30 \\
\hline & VO2max & & & & .149 & $2.27 *$ & .51 & 1.98 \\
\hline
\end{tabular}

$* p<.05 ; * * p<.01 ; * * * p<.001$

VO2max = consumo máximo oxigeno; \% MG= porcentaje de masa grasa; FPT= flexión profunda de tronco; $D-W=D$ urbin $-W$ atson; $T=$ tolerancia; $F I V=$ factor de inflación de la varianza.

Tal y como se puede observar en la tabla 3, los análisis indican que las variables predictoras tienen valores significativos en la predicción de las puntuaciones del autoconcepto académico, siendo incluidos en el modelo el consumo máximo de oxígeno y la flexión profunda de tronco. También sobre las puntuaciones del autoconcepto social, estando incluidas las variables porcentaje de masa grasa y la flexión profunda de tronco. A su vez, las variables consumo máximo de oxígeno y porcentaje de masa grasa predicen significativamente las puntuaciones del autoconcepto emocional y autoconcepto físico, al cual hay que añadir el tiempo en recorrer 50 metros.

\section{Discusión}

El presente trabajo trata de determinar si el porcentaje de masa grasa y algunas variables de la condición física tienen relación y son predictoras de la percepción del autoconcepto multidimensional en una muestra de adolescentes, usando para ello análisis de correlación y regresión lineal múltiple. Este objetivo tiene una gran relevancia en estas edades dadas las implicaciones que el autoconcepto posee en el desarrollo personal y social de los adolescentes, siendo un constructo muy estudiado en las últimas décadas. En esta etapa, son numerosos los cambios a nivel físico, emocional o académico que suceden, los cuales pueden estar influidos por cómo sienten y se perciben (Esnaola et al., 2008; Goñi, FernándezZabala e Infante, 2012; Greenleaf, Petrie y Martin, 2010).

Respecto a los análisis descriptivos efectuados, se puede observar que las puntuaciones medias obtenidas en el Cuestionario Autoconcepto Forma 5 (AF5- García y Musitu, 2001) se aproximan a las de otras investigaciones con poblaciones de edad similar, siendo las correspondientes al autoconcepto social y familiar las más elevadas (Jiménez, 2011; Malo, Bataller, Casas, Gras y González, 2011). Además, los porcentajes de masa grasa, así como las medidas de condición física se ajustan, en general, a los valores medios considerados para población adolescente, lo que permite considerar la muestra de este trabajo cercana a los perfiles mostrados en otras investigaciones (Moreno et al., 2006; Ortega et al., 2011).

Los análisis efectuados satisfacen los objetivos de la investigación, indicando que existe una relación significativa entre los indicadores de condición física, así como el porcen- 
taje de masa grasa, y las diferentes dimensiones del autoconcepto estudiadas. En concreto, las que poseen un mejor índice de correlación son las referidas al autoconcepto social, emocional y físico, aunque los análisis de regresión indican valores relevantes del porcentaje de la varianza explicada únicamente para los factores emocional y físico. Según Cohen (1988), los niveles de correlación obtenidos son moderados o bajos, aunque se puede considerar importante dado el carácter multifactorial de los modelos que suelen explicar los fenómenos psicosociales.

Los resultados encontrados se sitúan en la línea de otros que señalan la existencia de relaciones positivas entre la condición física y la percepción del autoconcepto en adolescentes. Las conclusiones de estos estudios ponen de relieve los vínculos existentes entre factores como la fuerza o la capacidad cardiorrespiratoria con medidas generales de autoconcepto y otras específicas como el autoconcepto físico, intelectual o social (Guillén y Ramírez, 2011; Petty, Davis, Tkacz, Young-Hyman y Waller, 2009; Sherrill, Holguin y Caywood, 1989; Strong et al., 2005; Vedul-Kjelsäs, Sigmundsson, Stensdotter y Haga, 2012; Velez et al., 2010). En la presente investigación, aunque la velocidad, la flexión profunda de tronco y el salto horizontal han correlacionado significativamente con las diferentes dimensiones del autoconcepto estudiado, el consumo máximo de oxígeno es la medida que más relevancia ha tenido. Este aspecto coincide con los trabajos mencionados, que han manifestado la importancia de los indicadores de tipo cardiorrespiratorio en el estudio de estas autopercepciones.

El porcentaje de masa grasa ha sido otra de las variables que ha obtenido una alta asociación con las medidas de autoconcepto evaluadas. Estos resultados se encuentran en la línea señalada por otros autores, que indican la existencia de estas relaciones, tanto con percepciones más generales del concepto personal como otras más específicas, como la académica, social, emocional y física (O’Dea, 2006; Zeller, Reiter-Purtill, Ratcliff, Inge y Noll, 2011). Diversos autores han puesto de manifiesto que el sobrepeso está vinculado a una peor percepción de la habilidad e imagen física, afectando a su bienestar psicológico y estado de ánimo, lo que puede afectar a la evaluación en otros ámbitos de sus vidas (Colella, Morano, Robazza y Bortoli, 2009; Goñi y Rodríguez, 2007; Goñi, Rodríguez y Ruiz de Azúa, 2004).

Gran parte de los estudios que han relacionado la condición física y la composición corporal con el autoconcepto se

\section{Referencias}

Annesi, J. J. (2010). Relationship of physical activity and weight loss in women with Class II and Class III obesity: Mediation of exerciseinduced changes in tension and depression. International Journal of Clinical and Health Psychology, 10(3), 435-444

Carraro, A., Scarpa, S. y Ventura, L. (2010). Relationships between physical self-concept and physical fitness in italian adolescents. Perceptual and Motor Skills, $110(2)$, 522-530.

Castillo, I. y Molina-García J. (2009). Adiposidad corporal y bienestar psicológico: efectos de la actividad física en universitarios de Valencia, España. Revista Panamericana de Salud Pública, 26(4), 334-340. han centrado, sobre todo, en el autoconcepto físico. En ellos se ha observado la asociación significativa con el concepto físico general, así como otras dimensiones específicas como condición física, fuerza o habilidad física, también sobre población adolescente (Crocker et al., 2006; Greenleaf et al., 2010; Lubans y Cliff, 2011; Morano, Colella, Robazza, Bortoli y Capranica, 2011). Además, estos autores ponen de relieve que los factores relacionados con la condición física son medidas más fiables para valorar aspectos del autoconcepto que la mera práctica de actividad física, lo que debe ser tenido en cuenta a la hora de diseñar programas de intervención que busquen mejorar el nivel de estas percepciones en poblaciones de estas edades.

Aunque en el estudio de Guillén y Ramírez (2011), en el que se analizan aspectos similares a los del presente trabajo, se obtienen resultados significativos, los autores consideran que son asociaciones débiles y no ofrecen conclusiones robustas sobre este fenómeno. Las diferencias con la presente investigación pueden deberse a la edad de los participantes, con una media de 11 años, y a los cambios que se producen en la adolescencia a nivel social, físico o cognitivo. Modificaciones en la imagen personal obliga a reformular el autoconcepto, contribuyendo paulatinamente a su consolidación (Esnaola, 2005). A medida que avanza la adolescencia, la fuerza de los estereotipos genera, posiblemente, una mayor dependencia del aspecto físico, lo que hay que considerar cuando se reflexiona sobre este tipo de aspectos (Dieppa, Guillén, Machargo y Luján, 2008).

En base a los resultados obtenidos, se puede decir que la condición física está relacionada con el autoconcepto multidimensional, prediciendo significativamente las dimensiones académica, social, emocional y física, en la muestra de este trabajo. No obstante, hay que señalar que el autoconcepto emocional y físico han sido las medidas mejor explicadas. Como en la mayoría de los trabajos de tipo transversal, sería interesante realizar estudios con diseños longitudinales que puedan ofrecer una perspectiva más amplia sobre los cambios producidos en la adolescencia. En cualquier caso, esta investigación pone de manifiesto la importancia que tiene las variables vinculadas a la condición física sobre estas autopercepciones, lo que debe ser valorado adecuadamente dado el impacto que tiene el concepto personal el desarrollo de los adolescentes.

Chaddock, L., Hillman, C.H., Pontifex, M.B., Johnson, C., Raine, L.B. y Kramer, A.F. (2012). Childhood aerobic fitness predicts cognitive performance one year later. Journal of Sports Sciences, 30(5), 421-430.

Cohen, J. (1988). Statistical power analysis for the behavioural sciences ( $\left.2^{\mathrm{a}} \mathrm{ed}.\right)$. Hillsdale, N. J.: Erlbaum.

Colella, D., Morano, M., Robazza, C. y Bortoli, L. (2009). Body image, perceived physical ability, and motor performance in nonoverweight and overweight Italian children. Perceptual and Motor Skills, 108(1), 209-218.

Crocker, P. R. E., Sabiston, C. M., Kowalski, K. C., McDonough, M. H. y Kowalski, N. (2006). Longitudinal assessment of the relationship be- 
tween physical self-concept and health-related behavior and emotion in adolescent girls. Journal of Applied Sport Psychology, 18(3), 185-200.

Cubo, S. (2011). Investigación cuasiexperimental. En S. Cubo, B. Martín y J.L. Ramos (Eds.), Métodos de investigación y análisis de datos en ciencias sociales $y$ de la salud (pp. 235-328). Madrid: Pirámide.

Dieppa, M., Guillén, F., Machargo, J. y Luján, I. (2008). Autoconcepto general y físico en jóvenes españoles y brasileños que practican actividad física vs. no practicantes. Revista de Psicología del Deporte, 17(2), 221-339.

Dunton, G. F., Schneider, M. y Cooper, D. M. (2007). An investigation of psychosocial factors related to changes in physical activity and fitness among female adolescents. Psychology and Health, 22(8), 929-944.

Dunton, G. F., Schneider, M., Graham, D. J. y Cooper, D. M. (2006). Physical activity, fitness, and physical self-concept in adolescent females. $P e-$ diatric Exercise Science, 18, 240-251.

Eisenmann, J.C., Wickel, E. E., Welk, G. J. y Blair, S. N. (2005). Relationship between adolescent fitness and fatness and cardiovascular disease risk factors in adulthood: the Aerobics Center Longitudinal Study (ACLS). American Heart Journal, 149(1), 46-53.

Esnaola, I. (2005). Desarrollo del autoconcepto durante la adolescencia y principio de la juventud. Revista de Psicología General y Aplicada, 58(2), 265-277.

Esnaola, I., Goñi, A. y Madariaga, J. M. (2008). El autoconcepto: perspectivas de investigación. Revista de Psicodidáctica, 13(1), 179-194.

Esnaola, I., Rodríguez, A. y Goñi, E. (2011). Propiedades psicométricas del cuestionario de Autoconcepto AF5. Anales de Psicología, 27(1), 109-117.

Eurofit (1993). Eurofit Tests of Physical Fitness ( $2^{\mathrm{a}}$ ed.). Strasbourg: Committee of Experts on Sports Research.

Ferreira, I., Twisk, J. W., Stehouver, C. D., Van Mechelen, W., Kemper, H. C. y Stehouwer, C. (2005). The metabolic syndrome, cardiopulmonary fitness, and subcutaneous trunk fat as independent determinants of arterial stiffness: the Amsterdam growth and health longitudinal study. Archives of International Medicine, 165(8), 75-82.

Franklin, J., Denyer, G., Steinbeck, K. S., Caterson, I.A. y Hill, A. J. (2006). Obesity and risk of low selfesteem: a statewide survey of Australian children. Pediatrics, 118(6), 2481-2487.

García, F. y Musitu, G. (2001). Autoconcepto Forma 5. AF5. Manual. Madrid: TEA.

García-Artero, E., Ortega, F. B., Ruiz, J. R., Mesa, J.L., Delgado, M., González-Gross, M., García-Fuentes, M., Vicente-Rodríguez, G., Gutiérrez, A. y Castillo, M. J. (2007). El perfil lipídico-metabólico en los adolescentes está más influido por la condición física que por la actividad física (estudio AVENA). Revista Española de Cardiología, 60(6), 581-588.

George, J. D., Fisher, A. G. y Vehrs, P. R. (2007). Tests y pruebas físicas (4 ed.). Barcelona: Paidotribo.

Goñi, E., Fernández-Zabala, A. e Infante, G. (2012). El autoconcepto personal: diferencias asociadas a la edad y al sexo. Aula Abierta, 40(1), 3950 .

Goñi, E. y Infante, G. (2010). Actividad físico-deportiva, autoconcepto físico y satisfacción con la vida. European Journal of Education and Psychology, 3(2), 199-208.

Goñi, A. y Rodríguez, A. (2007). Variables associated with the risk for eating disorders in adolescence. Salud Mental, 30(4), 16-23.

Goñi, A., Rodríguez, A., y Ruiz de Azúa, S. (2004). Bienestar psicológico y autoconcepto físico en la adolescencia y juventud. Psiquis, 25(4), 141151.

Greenleaf, C. A., Petrie, T. A. y Martin, S. B. (2010). Psychosocial variables associated with body composition and cardiorespiratory fitness in middle school students. Research Quarterly for Exercise and Sport, 81( 3), S65S74.

Guillén, F. y Ramírez, M. (2011). Relación entre el autoconcepto y la condición física en alumnos del Tercer Ciclo de Primaria. Revista de Psicología del Deporte, 20(1), 45-59.

Hair, J. F., Anderson, R. E., Tatham, R. L. y Black, W. (1999). Análisis multivariante $\left(5^{\mathrm{a}}\right.$ ed.). Madrid: Prentice Hall.

Jiménez, T. I. (2011). Autoestima de riesgo y protección: Una mediación entre el clima familiar y el consumo de sustancias en adolescentes. Psychosocial Intervention, 20(1), 53-61.

Lubans, D. R. y Cliff, D. (2011). Muscular fitness, body composition and physical self-perception in adolescents. Journal of Science and Medicine in Sport, 14(3), 216-221.
Malo, S., Bataller, S., Casas, C., Gras, M. E. y González, M. (2011). Análisis psicométrico de la escala multidimensional de autoconcepto AF5 en una muestra de adolescentes y adultos de Cataluña. Psicothema, 23(4), 871-878.

Martín, B. (2011). Técnicas e instrumentos de recogida de información. En S. Cubo, B. Martín y J.L. Ramos (Eds.), Métodos de investigación y análisis de datos en ciencias sociales y de la salud (pp. 387-407). Madrid: Pirámide.

Martínez, E. . (2011). Pruebas de aptitud física (2 ${ }^{\mathrm{a}}$ ed.). Barcelona: Paidotribo.

Martins, C. L., Silva, F., Gaya, A. R., Aires, L., Ribeiro, J. C. y Mota, J (2010). Cardiorespiratory fitness, fatness, and cardiovascular disease risk factors in children and adolescents from Porto. European Journal of Sport Science, 10(2), 121-127.

Morano, M., Colella, D., Robazza, C., Bortoli, L. y Capranica, L. (2011) Physical self-perception and motor performance in normal-weight, overweight and obese children. Scandinavian Journal of Medicine and Science in Sports, 21(3), 465-473.

Moreno, L. A., Mesana, M. I., González-Gross, M., Gil, C. M., Fleta, J., Warnberg, J., Ruiz, J. R., Sarria, A., Marcos, A. y Bueno, M. (2006). Anthropometric body fat composition reference values in Spanish adolescents. The AVENA Study. European Journal of Clinical Nutrition, 60(2), 191-196.

Moreno, J. A., Moreno, R. y Cervelló, E. (2009). Relación del autoconcepto físico con las conductas de consumo de alcohol y tabaco en adolescentes. Adicciones, 21(2), 147-154

Mota, J., Santos, R. M., Silva, P., Aires, L., Martins, C. y Vale, S. (2012). Associations between self-rated health with cardiorespiratory fitness and obesity status among adolescent girls. Journal of Physical Activity and Health, 9(3), 378-381.

Ortega, F. B., Artero, E. G., Ruiz, J. R., España-Romero, V., JiménezPavón, D., Vicente-Rodriguez, L.A., Moreno, G., Manios, Y., Béghin, L., Ottevaere, C., Ciarapica, D., Sarri, K., Dietrich, S., Blair, S.N., Kersting, M., Molnar, D., González-Gross, M., Gutiérrez, A., Sjöström, M. y Castillo, M. J. (2011). Physical fitness levels among European adolescents: the HELENA study. British Journal of Sports Medicine, 45(1), 20 29.

O'Dea, J. A. (2006). Self-concept, self-esteem and body weight in adolescent females. A three-year longitudinal study. Journal of Health Psychology, 11(4), 599-611.

Padilla-Moledo, C., Castro-Piñero, J., Ortega, F.B., Mora, J., Márquez, S., Sjöström, M. y Ruiz, J. R. (2012). Positive health, cardiorespiratory fitness and fatness in children and adolescents. European Journal of Public Health, 22(1), 52-56.

Pardo, A. y Ruiz, M. A. (2005). Análisis de datos con SPSS 13 Base. Madrid: McGraw Hill.

Petty K. H., Davis C. L., Tkacz J., Young-Hyman D. y Waller, J. (2009). Exercise effects on depressive symptoms and self-worth in overweight children: A randomized controlled trial. Journal of Pediatric Psychology, 34(9), 929-939.

Pontifex, M. B., Raine, L. B., Johnson, C. R., Chaddock, L., Voss, M. W. Cohen, N. J., Kramer, A. F. y Hillman, C. H. (2011). Cardiorespiratory fitness and the flexible modulation of cognitive control in preadolescent children. Journal of Cognitive Neuroscience, 23(6), 1332-1345.

Ramírez-Lechuga, J., Femia, P., Sánchez-Muñoz, C. y Zabala, M. (2011). La actividad física en adolescentes no muestra relación con el consumo máximo de oxígeno. Archivos de Medicina del Deporte, 28(142), 103-112.

Ruiz, J. R., Castro-Piñero, J., Artero, E. G., Ortega, F. B., Sjöström, M., Suni, J. y Castillo, M. J. (2009). Predictive validity of health-related fitness in youth: a systematic review. British Journal of Sports Medicine, 43(12) 909-923.

Shavelson, R. J., Hubner, J. J. y Stanton, J. C. (1976). Self-concept: validation of construct interpretations. Review of Educational Research, 46(3), $407-$ 441.

Sherrill, C., Holguin, O. y Caywood, A., J. (1989). Fitness, attitude toward physical education, and self-concept of elementary school children. Per ceptual and Motor Skills, 69(2), 411-414.

Slutzky, C. B. y Simpkins, S. D. (2009). The link between children's sport participation and selfesteem: exploring the mediating role of sport selfconcept. Psychology of Sport and Exercise, 10(3), 381-389

Strong, W. B., Malina, R. M., Blimkie, C. J., Daniels, S. R., Dishman, R. K., Gutin, B, Hergenroeder, A. C., Must, A., Nixon, P. A., Pivarnik, J. M., 
Rowland, T., Trost, S. y Trudeau, F. (2005). Evidence based physical activity for school-age youth. Journal of Pediatrics, 146(6), 732-737.

Vedul-Kjelsäs, V., Sigmundsson, H., Stensdotter, A. K. y Haga, M. (2012). The relationships between motor competence, physical fitness and selfperception in children. Child: Care, Health and Development, 38(3), 394402.

Velez, A., Golem, D. L y Arent, S. M. (2010). The impact of a 12-week resistance training program on strength, body composition, and self- concept of Hispanic adolescents. Journal of Strength and Conditioning Research, 24(4), 1065-1073.

Zeller M. H., Reiter-Purtill, J., Ratcliff, M. B., Inge, T. H. y Noll, J. G. (2011). Two-year trends in psychosocial functioning after adolescent Roux-en-Y gastric bypass. Surgery for Obesity and Related Disease, 7(6), 727732.

(Artículo recibido: 8-8-2012; revisión recibida: 29-11-2013; aceptado: 4-5-2013) 